\title{
Research on Superficial Area Measurement Method of Irregular Object Based on Image Processing
}

\author{
$\mathrm{Yu} Y i^{1, \mathrm{a}}$ \\ ${ }^{1}$ Hunan University Of Humanities, Science And Technology, Loudi, \\ Hunan , 417700, China \\ a351863292@qq.com
}

\begin{abstract}
: every field needs to face with problem on superficial area measurement of irregular object, how to quickly, effectively and accurately measure the superficial area of irregular object has appositive effect on increasing economic benefit, so it attracts universal attention. With the development and application of computer digital image technology, it has obtained excellent achievement in the different fields. For this, this paper puts forward one kind of superficial measurement method based of irregular object based on image processing, it separates target area and background area through collecting the gray level image being measured and combining with threshold image segmentation method. And then it uses boundary following to obtain information such as boundary point of target area as well as tracking direction and pixel count of boundary point, so it can well measure superficial area of irregular object. This method has advantage of simple operation and high accuracy, so it has higher application and popularization values.
\end{abstract}

Key Words: image processing; superficial area measurement; irregular object; method; research

\section{Introduction}

With the development of modern industry, there are more and more irregular objects are appearing, the traditional measurement content and way in the past can not meet its demand, the appearance of object and data acquisition has become to be one new and important research direction in the measurement field, accurate measurement on superficial area of irregular object has obtained universal attention. For example, the shape of metal sheet or cloth is irregular after cutting, it usually needs to calculate its actual area, the shape of leather is also irregular, and accurately calculating leather area is beneficial to make active cost accounting, improve production process operation etc, so it is very necessary to make accurate measurement on leather area.

\section{Overview to digital image processing}

Digital image processing is also regarded as computer image processing; it means the process of converting image signal into digital signal and completely using computer to make processing. This technology is appeared as early as in the 1950s, and it has become to be one subject up to 1960s. In the process of computer processing, image is divided into one small area and each area represents one pixel. Digital image can be expressed by 2-D matrix, of which, one point on the matrix is one pixel, and it can describe the whole image by learning about gray level of pixel. 
Here the gray level means the color level of point in the image, the range varies from 0 to 255 , of which the white depth is 255 and black is 0 .

The digitized image is the orderly data in the computer; it can make processing on digital image according to demand, but all needs to undergo image data input, processing and output.

With the development of computer technology and digital camera shooting technology, machine vision technology has obtained quick development in recent years. While there is more and more work on superficial outline, physical dimension and measurement on free-form surface, the accuracy requirement is becoming higher and higher, which makes image processing technology obtain popularization with wide range. On realization of image processing technology, it uses digital image processing technology to automatically calculate its superficial area through the video data of measured object collected by CCD camera, it is one kind of effective measure with low cost, non-contact, high precision, little error, flexible application and quick measurement speed, while the actual area represented by each pixel even can reach grade of square millimeter. It can be seen that using image measurement technology to make accurate measurement on irregular object has certain advantage.

\section{Image measurement principle}

Image measurement firstly needs to use optical system to make imaging of measured object physical dimension on CCD photosensitive surface by certain accurate multiplying power, and then make AS conversion on CCD output, these content is transmitted to computer to make relevant processing and realize measurement on object.

From this it can be seen that before image measurement, it needs to make calibration on object, which means making processing on one object with know dimension and get multiplying power relation between object and pixel, it is the pixel equivalent coefficient. Its calculation formula is as follows:

$$
\mathrm{D}=\varepsilon * \mathbb{N}(1)
$$

In equation (1), D represents the geometric parameter of object waiting to be measured, $N$ is the pixel number of object waiting to be measured, $\varepsilon=\frac{\mathrm{s}}{\mathrm{M}}$ is the pixel equivalent coefficient. Of which, $\mathrm{S}$ is the geometric parameter of calibrated object; $\mathrm{M}$ is the pixel number of measured calibrated object.

\section{Design and realization of image measurement system}

After learning about principle of image measurement, if it wants to complete superficial area measurement of irregular object, it needs to make design on image measurement system to really achieve area measurement. While image measurement system mainly includes hardware and software, all needs to make design and make on 
supporting facility according to relevant requirement, only this can it guarantee the accuracy of superficial area measurement for irregular object.

Hardware system of image measurement is composed of sub-system of lighting, sub-system of image collection and sub-system of image processing. Here the sub-system of lighting mainly includes light source and light source controller, sub-system of image collection includes optical lenses, CCD, image collection card etc. Sub-system of image processing is mainly computer. The designed image measurement hardware structure according to the above-mentioned requirement is indicated by the following figure:

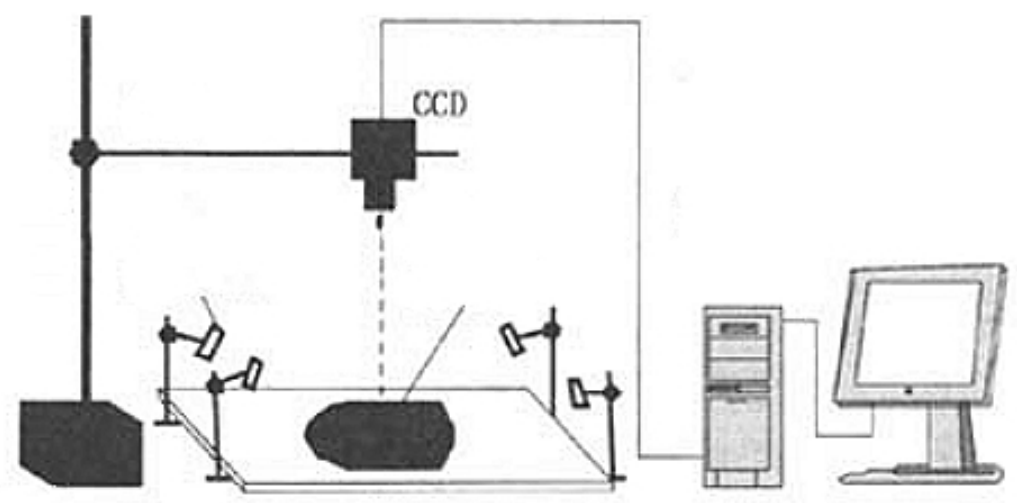

Figure 1 Diagrammatic sketch of hardware system structure of image measurement

From the above figure 1, it can be seen that it needs certain supporting facilities except for relevant parts of image collection sub-system, image processing sub-system, which is mainly connection facilities such as object table and pillar etc. The function of object table is to bear the measured object and unify the background based on measured object. The connection facilities is existed for the convenient application of hardware facility, including: prevent slide bar, system pillar and seat of camera. From figure 1, it can be seen that slide bar can be flexibly moved upwards and downwards to adjust object distance, the camera cal also slide on slide bar left and right to adjust position of camera, finally it is stabilized on the slide bar, make lens $\backslash$ face parallel to object table, place the measured object below than camera lens to possibly guarantee center of surface of object waiting to be measured and camera center in the same perpendicular bisector, the seat mainly has support function.

Software design of image measurement system uses design method of object procedure as instruction, its design target is quick and accurate measurement on irregular object, it uses Visual $\mathrm{C}++6.0$ as development platform. In the realization process of software system, it divides procedures into collection module, image processing module, data management, calculation module and interface design module etc according to function difference.

Image collection module is mainly responsible for image collection, transmission and storage in the computer. Image processing module is core module of image 
measurement software system, in order to count the pixel number of irregular object, it needs to make complicated processing, its process is indicated by the following figure 2. The main functions of data management and calculation module is to realize information exchange between operator and computer, correctly read operation of operators, which is to input information and accurately present data by computer processing in front of operators. Meanwhile, it needs to store the required data into computer and guarantees smooth transmission between data and information in computer software part. Interface design module is composed of interface appearance design and special function.

Image measurement software system can realize functions such as system calibration, area measurement, data check, deletion, print, result indication and output etc, which can better meet requirements in the actual application, so it has certain practice and application value.
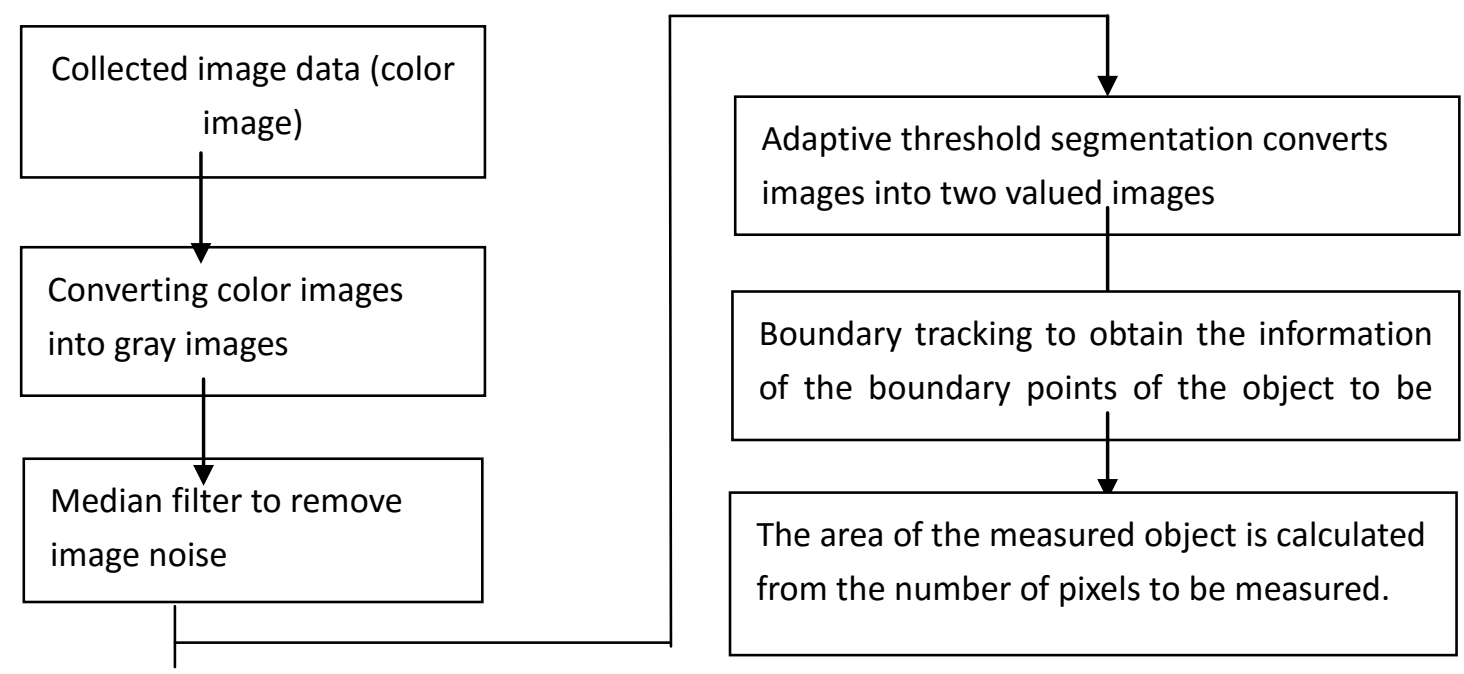

Figure 2 Diagrammatic sketch of image processing procedure

\section{Surface measurement method of irregular object based on image processing}

Because it measures the surface parameter of irregular object, it mainly uses collected image to obtain the overall outline information of regional image. Through image processing, it can obtain relevant image boundary information and extract the overall outline of measured object so as to measure its superficial area. Of which, the usual image segmentation methods is threshold segmentation, which means making division according to gray level on both sides of image boundary. Firstly, it chooses medium gray level as initial threshold value $\mathrm{T}_{0}$ as division standard and divide the whole image into different regions $G_{1} 、 G_{2}$, it uses relevant calculation formula to calculate the average gray level $\mu_{1}$ and $\mu_{2}$ by using relevant calculation formula, and then it calculates the new threshold through formula 2:

$$
\mathrm{T}_{\mathrm{j}+1}=\frac{1}{2}\left(\mu_{1}+\mu_{2}\right)
$$


Continually to repeat the above-mentioned calculation steps until difference between $T_{j+1}$ and $T_{j}$ is smaller than certain given value. Because the gray level of image is positive number, so the difference of 2 gray levels is also integer, in order to guarantee its universal property, the given value is better made as 1 .

After dividing measured target area and background through image processing, it needs to calculate regional area according to boundary tracking way. When using boundary tracking to extract image regional outline, it needs to record grade of each boundary point and search direction, finally it gets the area of target region. Its detailed process is indicated as follows:

Firstly, it makes tracking on 8 adjacent boundaries on target area according to counter-clockwise direction and calibrates it on boundary point from bigger to smaller; the biggest grade is the total of image boundary pixel. Secondly, it combines with boundary point grade and regards route from the front boundary point ( $\mathrm{P}-1)$ to the current boundary point $(\mathrm{P})$ as the front vector of current boundary point. The route from the corresponding boundary point to the next boundary point is the backward vector, the vector direction and vector value adopts eight direction code of Wang Houda, which is indicated by the following figure 3 :

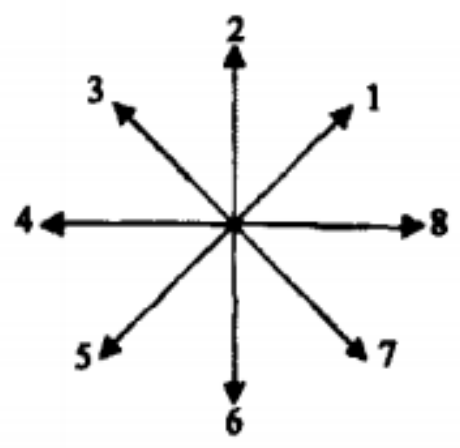

Figure 3 Eight direction code

In order to make it convenient for superficial area calculation, it needs to make sequencing on the obtained boundary point by sequence from upwards to downwards, and from left to right, and then calculates the total number of pixel within boundary. If the current boundary point meets condition that the pixel on the right is boundary point, then it calculates the pixel number between current boundary point and then next boundary point adjacent to it, which is $X_{+1}-X_{i}-1$, of which, $X_{i}$ represents the train value of front boundary point, $X_{i+1}$ is the train value between current boundary point and the next boundary point adjacent to it. Through test, it finds that the condition for the pixel on the right of current boundary point is the internal boundary point is as follows: $\mathrm{nv} \neq 8$, at the same time $\mathrm{pv}=5$, or $\mathrm{pv}<3$ and $|\mathrm{pv}-\mathrm{nv}|>4$, or $\mathrm{pv}$ $>5$ and $|p v-n v|<4$. And the final the superficial area measurement of irregular object is indicated as follows: 
Area $=\left(\frac{\text { Total number of boundary pixels }}{2}+\right.$ Total number of pixels in the border $) \times$ pixel

equivalent coefficient

Through the above superficial area measurement equation of irregular object, we can easily get the demand area data, for example, it makes superficial area measurement on 4 pieces of irregular leather, of which, the total boundary pixel of leather 1 is 13888 , and the pixel total number within boundary is 121465 , the boundary pixel of leather 2 and the pixel total number within boundary is respectively 2092 and 240106, leather 3 is 2324, 299678, leather 4 is 1999 and 253969. Their measurement result of superficial area is indicated by the following table 1 :

Table 1 Measurement results $\quad\left(\mathrm{cm}^{2}\right)$ on superficial area of different irregular leather

\begin{tabular}{|c|c|c|c|c|}
\hline & Leather 1 & Leather 2 & Leather 3 & Leather 4 \\
\hline $\begin{array}{c}\text { total boundary } \\
\text { pixel }\end{array}$ & 1388 & 2092 & 2324 & 1999 \\
\hline $\begin{array}{c}\text { total pixel } \\
\text { within } \\
\text { boundary }\end{array}$ & 121465 & 240106 & 299678 & 253969 \\
\hline superficial area & 1476.287 & 2914.313 & 3635.641 & 3081.291 \\
\hline
\end{tabular}

It obtains gray level image of irregular object through image processing, and then it uses threshold segmentation method to divide target area and background area, then calculates region area through boundary tracking. It provides new method for superficial area measurement of irregular object, and precision of measurement result is as high as $\pm 1 \%$, the operation is very simple and so it has great application and practice prospect.

\section{References}

[1]Gonzalez R C， Woods R E. Digital Image Processing [M]. Translated by Ruan Qiuqi, Ruan Yuzhietc. Beijing: Publishing House of Electronics Industry, 2003

[2]Hong Ying, Dang Hongshe, Song Jinguo. Superficial Area Measurement Method of Irregular object Based on Image Processing [J].Computer Measurement and Control, 2009,17 (9): 1679-1681

[3]Dang Hongshe, Hong Ying, Guo Qin. Realization on Area Measurement of Irregular Object Based on Image Processing. Computer Measurement and Control, 2010,18 (7): 1507-1508,1511

[4]Zhang Yuanyuan. Research and Application on Projected Area of Irregular Object Based on Image Processing [D]. Master Thesis of Changchun University of Science 
and Technology, 2010: 13-31

[5]Ge Zhenhua, Chen Youguang. Regional Area Calculation Based on Boundary Tracking [J]. Computer Application and Software， 2008,25 (6): 239-274

[6]Liu Yi, Lai Xiaofeng. Discussion on Image Segmentation Technology Based on Boundary Test [J]. Practical Electronics， 2013 (16): 67,62

[7]Shi Gaoshan. Research on Measurement Method of Optical Crystal Non-linear Nature Based on Digital Processing Technology [D]. Master Thesis of Shandong University, 2010:6-8

[8]Zhang Yujin. Processing and Analysis on Digital Image [M].Beijing: Tsinghua University Publishing House, 2002

[9]He Bin.Visual C++Digital Image Processing [M].Beijing : Posts and Telecommunications Press, 2001 